\title{
Fault Diagnosis of Hydraulic Pumps Using PSO-VMD and Refined Composite Multiscale Fluctuation Dispersion Entropy
}

\author{
Fuming Zhou (iD, Xiaoqiang Yang, Jinxing Shen (D), and Wuqiang Liu \\ Field Engineering College of Army Engineering University, Nanjin 210007, China \\ Correspondence should be addressed to Jinxing Shen; 565423803@qq.com
}

Received 4 June 2020; Revised 14 July 2020; Accepted 23 July 2020; Published 24 August 2020

Academic Editor: Francisco Beltran-Carbajal

Copyright (c) 2020 Fuming Zhou et al. This is an open access article distributed under the Creative Commons Attribution License, which permits unrestricted use, distribution, and reproduction in any medium, provided the original work is properly cited.

\begin{abstract}
Multiscale fluctuation dispersion entropy (MFDE) has been proposed to measure the dynamic features of complex signals recently. Compared with multiscale sample entropy (MSE) and multiscale fuzzy entropy (MFE), MFDE has higher calculation efficiency and better performance to extract fault features. However, when conducting multiscale analysis, as the scale factor increases, MFDE will become unstable. To solve this problem, refined composite multiscale fluctuation dispersion entropy (RCMFDE) is proposed and used to improve the stability of MFDE. And a new fault diagnosis method for hydraulic pumps using particle swarm optimization variational mode decomposition (PSO-VMD) and RCMFDE is proposed in this paper. Firstly, PSOVMD is adopted to process the original vibration signals of hydraulic pumps, and the appropriate components are selected and reconstructed to get the denoised vibration signals. Then, RCMFDE is adopted to extract fault information. Finally, particle swarm optimization support vector machine (PSO-SVM) is adopted to distinguish different work states of hydraulic pumps. The experiments prove that the proposed method has higher fault recognition accuracy in comparison with MSE, MFE, and MFDE.
\end{abstract}

\section{Introduction}

Hydraulic pumps are the core components of the entire hydraulic system, which are known as the "heart" of the hydraulic system. They are typical high-speed rotating machines which are often in a state of high speed and high load, so they are more to be out of order than other construction machines. The faults of hydraulic pumps will affect the work of the entire hydraulic system and even cause huge economic losses and casualties. Therefore, the research of hydraulic pumps fault diagnosis has very important practical significance [1-4].

Analyzing vibration signals of mechanical equipment is a common method for fault diagnosis $[5,6]$. The failure mechanism of hydraulic pumps is complex and the fault features of vibration signals are weak. Traditional linear signal processing methods are difficult to extract the fault information effectively [7]. Many nonlinear signal processing methods such as empirical mode decomposition (EMD) [8], wavelet analysis [9], and local mean decomposition (LMD) [10] have defects such as terminal effect and mode mixing, so there is still a large space for improvement.
Variational mode decomposition (VMD) [11] is a new signal processing method, which is adopted to decompose the original signals into a specific number of intrinsic mode functions (IMFs). This method avoids terminal effect and has higher calculation efficiency, so it has attracted the attention of many scholars. Zan [12] applied VMD to decompose bearing vibration signals to identify different bearing faults, while Song [13] combined VMD and multiscale entropy method to complete fault diagnosis of gearboxes, and they all achieved good results. However, the two core parameters of VMD, the penalty factor $\alpha$ and the number of IMFK, need to be set artificially. If not selected properly, there may be defects such as mode mixing. In order to overcome this shortcoming, we try to adopt appropriate optimization algorithm to select the best parameters of VMD. Commonly applied optimization algorithms include genetic algorithm (GA) [14], ant colony optimization (ACO) [15], and particle swarm optimization (PSO) [16], where particle swarm optimization is the most diffusely applied. So, we decide to employ PSO to optimize $\alpha$ and $K$ to get the best combination of parameters. PSO-VMD is employed to 
process the original vibration signals of hydraulic pumps and the IMF components containing abundant fault information are screened for reconstruction to get denoised vibration signals of hydraulic pumps. These signals have less noise pollution and are more conducive to fault diagnosis of hydraulic pumps.

Entropy is a good method to detect the stability of complex signals. The commonly used entropy includes sample entropy (SE) $[17,18]$, permutation entropy (PE) $[19,20]$, and fuzzy entropy (FE) [21, 22]. Since single-scale analysis does not consider the inherent time fluctuations of complex signals, based on multiscale entropy (MSE) theory [23], SE, PE, and FE are upgraded to multiscale sample entropy (MSE) [24], multiscale permutation entropy (MPE) [25], and multiscale fuzzy entropy (MFE) [26]. However, the calculation efficiency of SE is low, and it is unstable when dealing with short time series [27]. PE does not consider the influence of the amplitude difference of a given signal, and it is sensitive to noise [28]. FE is more stable than SE [29], but its calculation efficiency is also low. In order to address the above problems, Rostaghi and Azami proposed a new method called dispersion entropy (DE) [30], while it is more stable and has higher computation efficiency. On the basis of $\mathrm{DE}$, consider the fluctuation of signals and fluctuation dispersion entropy (FDE) [31] were proposed and carried out multiscale expansion to obtain multiscale fluctuation dispersion entropy (MFDE) [32].

However, the traditional coarse-graining method does not consider the relationship between each coarse-grained time series, and as the scale factor increases, the entropy values will become unstable [33]. Gan et al. [34] proposed composite multiscale fluctuation dispersion entropy (CMFDE), proving that the sliding coarse-graining method can improve the stability of MFDE. Inspired by this, refined composite multiscale fluctuation dispersion entropy (RCMFDE) is proposed. It should be noted that the principles of RCMFDE and CMFDE are basically similar, with the same coarse-graining method. The difference of them is the calculation of entropy. RCMFDE adopts the refined composite method to calculate the result of entropy, while CMFDE calculates the mean entropy values of different coarse-grained time series as the final result. In the rest of this article, RCMFDE, CMFDE, and MFDE are compared.

Combining PSO-VMD and RCMFDE, a novel fault diagnosis method of hydraulic pumps based on PSO-VMD, RCMFDE, and PSO-SVM is proposed. First, PSO-VMD is adopted to process the original vibration signals of hydraulic pumps, and the sensitive IMFs are filtered out through the correlation coefficient method for reconstruction to obtain the denoised vibration signals, then the fault features are extracted by RCMFDE, and finally the fault feature vectors are input into PSO-SVM to complete the fault diagnosis of hydraulic pumps. PSO-VMD can effectively remove the impact of noise and highlight the fault features. RCMFDE is more stable and has a stronger ability to extract fault features in comparison with MFDE, MSE, and MFE. And SVM is a powerful supervised machine learning method with good generalization ability, which possesses obvious advantages in dealing with small sample classification problems $[35,36]$.
Based on the excellent performance of the above methods, the method proposed can accurately distinguish different work states of hydraulic pumps. The experiments show that the proposed method has higher fault recognition accuracy in comparison with MSE, MFE, and MFDE.

In conclusion, the core innovation of this paper is the application of entropy theory in the field of fault diagnosis. RCMFDE is proposed and applied to hydraulic pump fault diagnosis; meanwhile, optimized VMD is proposed to preprocess vibration signals so as to highlight fault features. The rest structure of this paper is as follows. Sections 2 and 3 mainly explain the principle of PSO-VMD and RCMFDE. Section 4 mainly explains the proposed method in detail. Then, the superiority of this method is reflected through experiments in Section 5. Conclusions are summarized in Section 6.

\section{PSO-VMD}

2.1. VMD. VMD is a novel signal processing method, which is adopted to decompose the original signal into several IMFs. In this section, we briefly summarize the steps of VMD algorithm. The detailed principle of VMD is shown in [11].

The core idea of VMD is to address the following constrained optimization problem:

$$
\min _{\left\{u_{k}\right\},\left\{\omega_{k}\right\}}\left\{\sum_{k}\left\|\partial_{t}\left[\left(\sigma(t)+\frac{j}{\pi t}\right) * u_{k}(t)\right] * e^{-j \omega_{k} t}\right\|_{2}^{2}\right\},
$$

where $u_{k}$ is each IMF; $\omega_{k}$ is the center frequency of each IMF; and $\delta(t)$ is the unit pulse signal.

In order to solve the constraint problem, a quadratic penalty term $\alpha$ and Lagrangian multipliers $\lambda$ are introduced to obtain the unconstrained optimization model shown in equation (2). The saddle point of equations (2) is found by alternately updating $u_{k}^{n+1}, \omega_{k}^{n+1}$, and $\lambda^{n+1}$, which is the optimal solution of the unconstrained optimization model:

$$
\begin{aligned}
L\left(\left\{u_{k}\right\},\left\{\omega_{k}\right\}, \lambda\right)= & \alpha \sum_{k}\left\|\partial_{t}\left[\left(\delta(t)+\frac{j}{\pi t}\right) \cdot u_{k}(t)\right] e^{-j \omega_{k} t}\right\|_{2}^{2} \\
& +\left\|f(t)-\sum_{k} u_{k}(t)\right\|_{2}^{2}+\left\langle\lambda(t), f(t)-\sum_{k} u_{k}(t)\right\rangle .
\end{aligned}
$$

The optimal solution is

$$
\begin{aligned}
\widehat{u}_{k}^{n+1}(\omega) & =\frac{\widehat{f}(\omega)-\sum_{i=1, i \neq k}^{K} \widehat{u}_{i}(\omega)+(\widehat{\lambda}(\omega) / 2)}{1+2 \alpha\left(\omega-\omega_{k}\right)^{2}}, \\
\omega_{k}^{n+1} & =\frac{\int_{0}^{\infty} \omega\left|\widehat{u}_{k}(\omega)\right|^{2} \mathrm{~d} \omega}{\int_{0}^{\infty}\left|\widehat{u}_{k}(\omega)\right|^{2} \mathrm{~d} \omega} .
\end{aligned}
$$

2.2. $P S O-V M D$. The effect of VMD is greatly affected by $K$ (the number of IMF) and $\alpha$. They need to be set in advance when decomposing signals. The traditional method is to 
observe the change of center frequency of each IMF component when $k$ values different degrees. If components with similar frequencies appear, the last $K$ is taken. This method is too subjectively affected to apply in operation. PSO is a widely applied optimization algorithm [16], which has good global search capabilities. Therefore, this paper employs PSO to optimize $K$ and $\alpha$ of VMD to access the best combination of parameters.

PSO-VMD algorithm needs to determine a fitness function, calculate the corresponding fitness value when the particle position is updated, and update it by comparing the fitness value of old and new particles. It can be seen from the above that entropy can well reflect the dynamic features of complex time series. PE is sensitive to noise, and the higher the $\mathrm{PE}$ value, the higher the noise content of the component, while the smaller it indicates that the component contains more fault information. Therefore, the mean permutation entropy (MPE) is taken as the fitness function in this paper. Each particle represents a combination of $K$ and $\alpha$, and calculate the mean PE value of $K$ IMFs obtained by origin signals after VMD decomposition. The minimum mean permutation entropy (MMPE) of all particles is the fitness value. By iteratively updating the particles to update the fitness value, the global best fitness value can be obtained, and the corresponding $K$ and $\alpha$ is the best combination of Kand $\alpha$. Figure 1 shows the flow chart of PSO-VMD and the steps can be summarized as follows:

(1) Set the initial parameters of PSO algorithm and take $[K \alpha]$ as the particle position to generate the initial population

(2) Calculate the MPE corresponding to the particle positions of the initial population and obtain the MMPE as the fitness value

(3) Update particles and calculate the MPE corresponding to each particle after update

(4) Compare and update the fitness value

(5) Iterative loop and output the best $[K \alpha]$

\section{RCMFDE}

3.1. FDE. For the nonlinear time series $X=\left\{x_{1}, x_{2}, \ldots, x_{N}\right\}$, the detailed calculation steps of FDE algorithm are as follows:

(1) $x_{i}(i=1,2 \ldots N)$ is mapped to some integer from 1 to $c$ by equations (5) and (6):

$$
y_{i}=\frac{1}{\sigma \sqrt{2 \pi}} \int_{-\infty}^{x_{i}} e^{\left(-(t-\mu)^{2} / 2 \sigma^{2}\right)} \mathrm{d} t
$$

where $\mu$ is expectation and $\sigma^{2}$ is variance, and

$$
z_{i}=R\left(c \cdot y_{i}+0.5\right)
$$

where $c$ is an integer and $R$ is the rounding function.

(2) The time series $Z$ is reconstructed as follows:

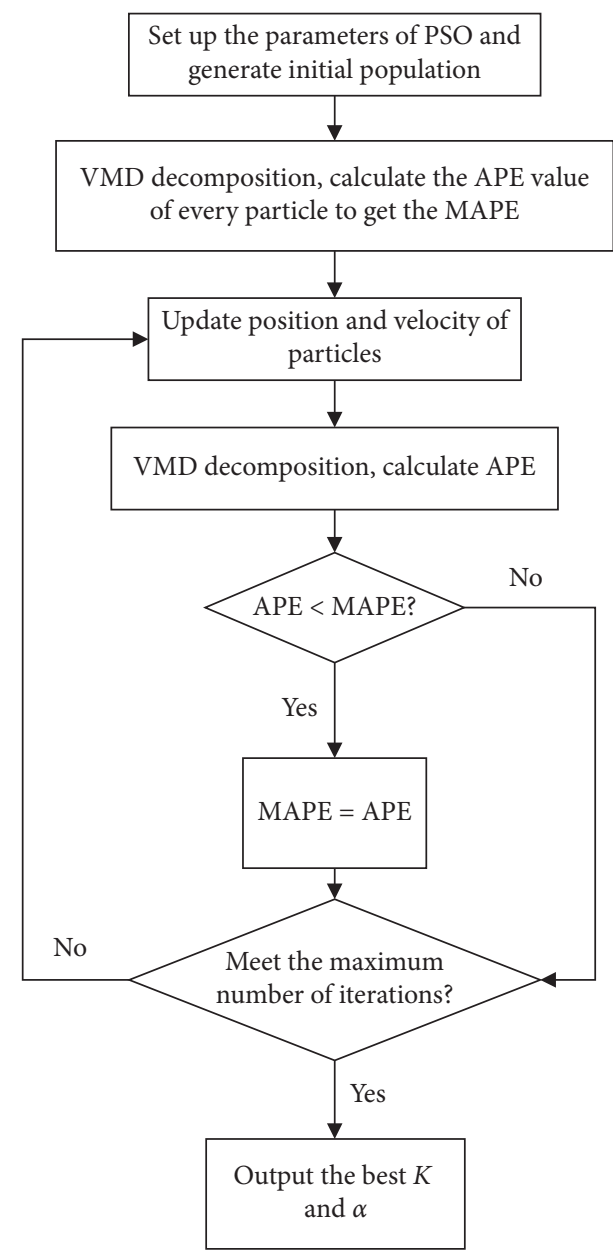

Figure 1: Flow chart of PSO-VMD.

$$
\begin{aligned}
Z_{j}^{m . \lambda . c} & =\left\{z_{j}, z_{j+\lambda}, \ldots, z_{(j+(m-1) \lambda)}\right\}, \\
j & =[1,2 \ldots N-(m-1) \lambda],
\end{aligned}
$$

where $m$ is the embedding dimension and $\lambda$ is the time delay.

(3) Transform $Z^{m, \lambda, c}$ to $Q^{m, \lambda, c}$ by

$$
Q_{j}^{m . \lambda . c}=\left\{z_{j+\lambda}-z_{j}+c, \ldots, z_{j+(m-1) \lambda}-z_{j+(m-2) \lambda}+c\right\} .
$$

Each time series $Q^{m, \lambda, c}$ has its corresponding fluctuation dispersion pattern $\pi_{v_{0} v_{1} \ldots v_{m-2}}(1 \leq v \leq 2 c-1)$ and $Q_{j, 1}^{m, \lambda, c}=v_{0}, Q_{j, 2}^{m, \lambda, c}=v_{1}, \ldots, Q_{j, m-1}^{m, \lambda, c}=v_{m-2}$. The probability of each pattern can be calculated by

$$
p\left(\pi_{v_{0} v_{1} \ldots v_{m-2}}\right)=\frac{\operatorname{Numbe}\left(\pi_{v_{0} v_{1} \ldots v_{m-2}}\right)}{N-(m-1) \lambda},
$$

where Numbe $\left(\pi_{v_{0} v_{1} \ldots v_{m-2}}\right)$ is the number of fluctuation dispersion patterns $\pi_{v_{0} v_{1} \ldots v_{m-2}}$. 


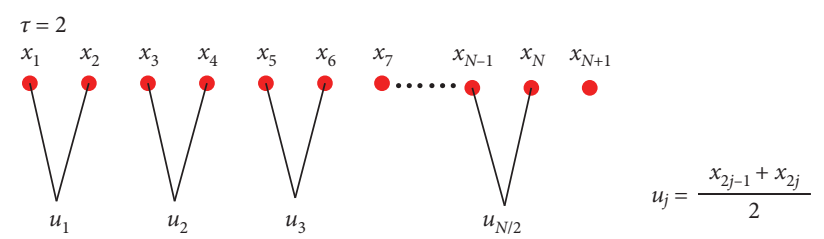

(a)
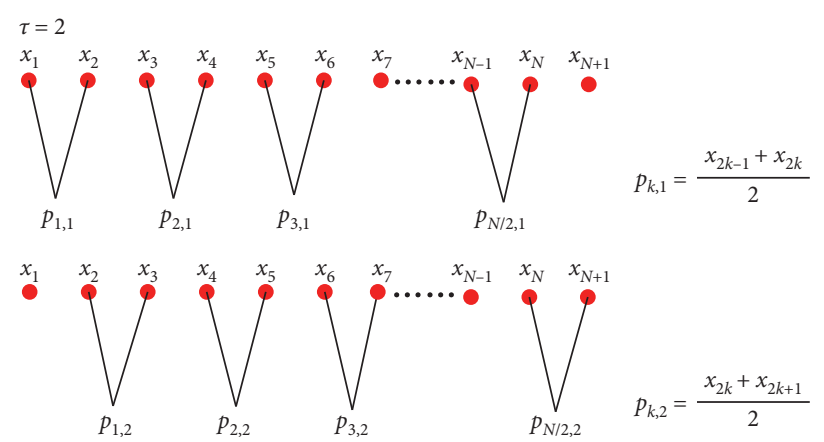

(b)

FIGURE 2: Two different coarse-graining methods. (a) The traditional coarse-graining method when $\tau=2$. (b) The sliding coarse-graining method when $\tau=2$.

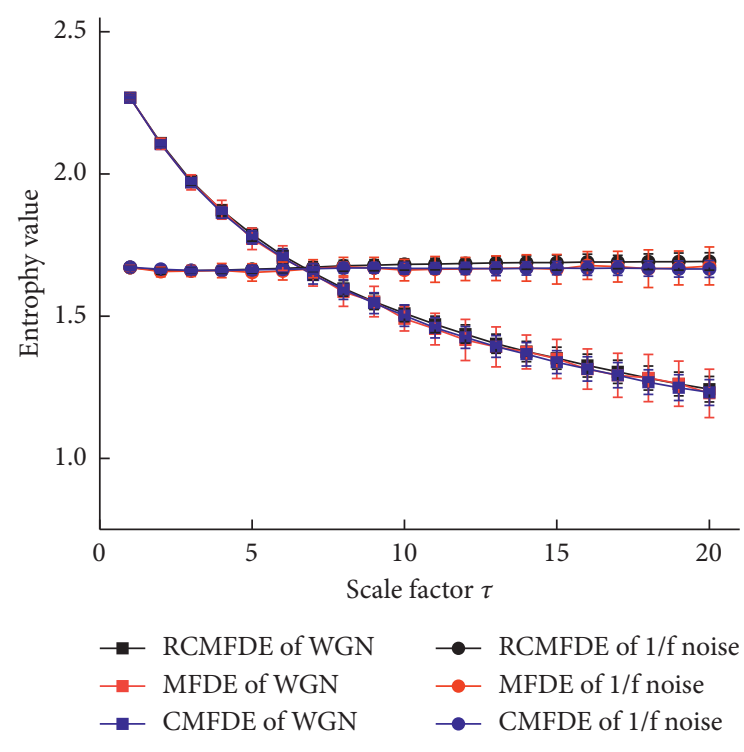

(a)

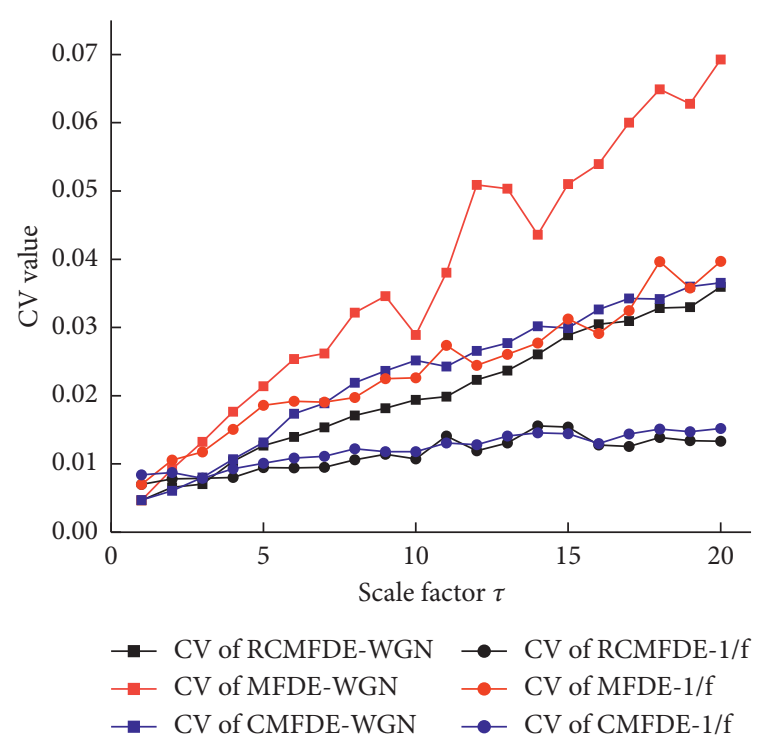

(b)

Figure 3: Comparison of RCMFDE, CMFDE, and MFDE. (a) Mean value and SD of WGN and 1/f noise using different methods. (b)CV of WGN and $1 / f$ noise using different methods.

(4) FDE is calculated by

$$
\operatorname{FDE}(X, m, c, \lambda)=-\sum_{\pi=1}^{(2 c-1)^{m-1}} p\left(\pi_{v_{0} v_{1} \ldots v_{m-2}}\right) \ln p\left(\pi_{v_{0} v_{1} \ldots v_{m-2}}\right) .
$$

\subsection{MFDE and RCMFDE}

(1) For $X=\left\{x_{1}, x_{2}, \ldots, x_{N}\right\}$, use equation (11) to coarse-graining it to get the processed time series $U=\left\{u_{1}, u_{2}, \ldots, u_{(N / \tau)}\right\}$. Figure $2($ a) shows the coarse-graining process:

$$
u_{j}=\frac{1}{\tau} \sum_{i=(j-1) \tau+1}^{j \tau} x_{i}
$$

where $\tau$ is scale factor.

(2) By calculating the FDE value of time series $U$ under the same parameters, the FDE values under different scale factors can be obtained.

The above coarse-graining method is currently the most commonly used data processing method for multiscale entropy. As shown in Figure 2, this method 

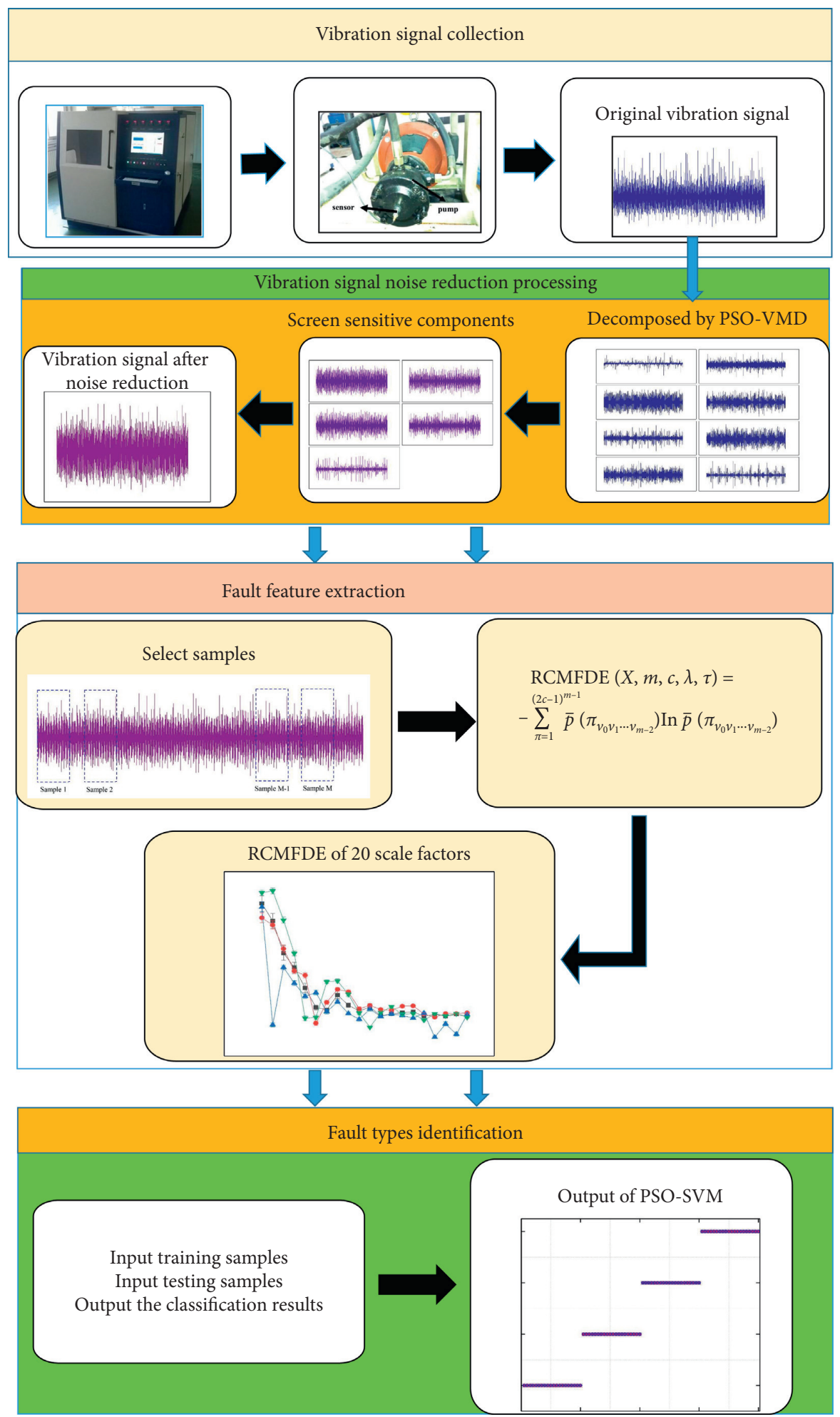

FIgURE 4: Flow chart of the proposed method.

does not take into account the relationship between the elements such as $x_{2}$ and $x_{3}, x_{4}$ and $x_{5}$, so it cannot guarantee maximum utilization of original time series data. And as $\tau$ increases, the coarse-grained time series gradually shortens, prone to unstable entropy, so this paper uses sliding coarse-graining data processing method to replace traditional coarsegraining method to calculate FDE values at different scales, which is called refined composite multiscale fluctuation dispersion entropy(RCMFDE).

(1) For $X=\left\{x_{1}, x_{2}, \ldots, x_{N}\right\}$, the $n$th coarse-grained time series is obtained by 


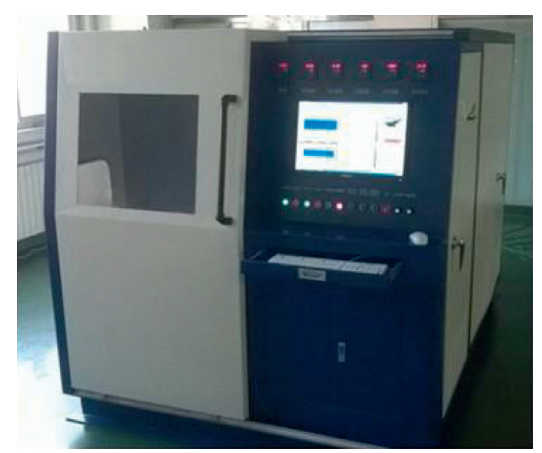

(a)

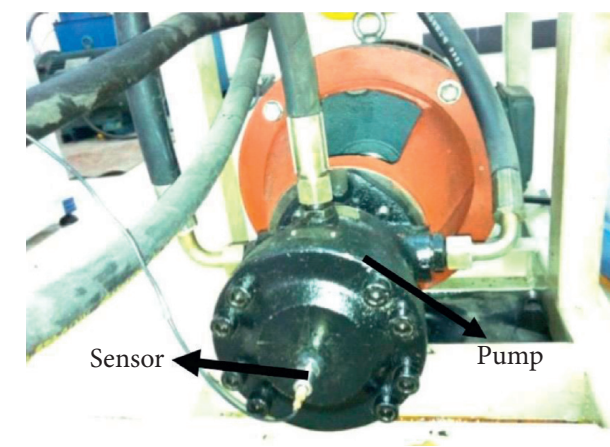

(b)

FIgURE 5: The experiment device. (a) The experiment platform of hydraulic pump. (b) Hydraulic pump and test device.

$$
p_{k, n}=\frac{1}{\tau} \sum_{i=n+\tau(k-1)}^{n+k \tau-1} x_{i}, 1<n<\tau, 1<k<N / \tau
$$

where $\tau$ is the scale factor.

(2) RCMDE is calculated by

$$
\begin{aligned}
\operatorname{RCMFDE}(X, m, c, \lambda, \tau)= & -\sum_{\pi=1}^{(2 c-1)^{m-1}} \bar{p}\left(\pi_{v_{0} v_{1} \ldots v_{m-2}}\right) \ln \bar{p} \\
& \cdot\left(\pi_{v_{0} v_{1} \ldots v_{m-2}}\right),
\end{aligned}
$$

where $\bar{p}\left(\pi_{v_{0} v_{1} \ldots v_{m-2}}\right)$ is the mean frequency of the same dispersion patterns in each coarse-grained time series.

The sliding coarse-graining processing method comprehensively considers all elements in original time series, avoids information omission in traditional coarse-graining processing method, and thus has better performance. Therefore, this paper adopts RCMFDE to extract fault feature information of hydraulic pumps.

3.3. Parameters of RCMFDE. The main parameters of RCMFDE are the embedding dimension $m$, the number of classes $c$, and the time delay $\lambda$. According to the principle of FDE, if $m=1$, the amplitude difference between adjacent elements cannot be measured, som $>1$. However, excessively large $m$ will reduce the computational efficiency. If $c$ is too small, some elements with huge amplitude differences will not be well distinguished. If $c$ is too large, the subtle amplitude differences may lead to different classifications. $\lambda$ has little effect on entropy value, which is usually $1[32,37]$. $m=2$ or 3 and $4<c<8$ are suggested in [30]. This paper is inspired by the parameters setting in $[30,31,34,37]$ when using DE and its improved forms to process noise simulation signals, biomedical signals, and rotating machinery vibration signals, respectively, and set $c=6, m=2$, and $\lambda=1$ finally.

3.4. Comparison of RCMFDE, CMFDE, and MFDE. In order to compare the performance of RCMFDE, CMFDE, and MFDE, simulation noise signals are adopted for experiments, in which CMFDE, MFDE, and RCMFDE have the same parameters, where $c=6, m=2$, and $\lambda=1$. Fifty groups of white noise and $1 / f$ noise signals are selected. The length of simulation noise signals is 2048. Figure 3 shows the simulation experiment results.

It is observed from Figure 3(a) that, with the increase of scale factor, the entropy value of white noise gradually decreases while the entropy value of $1 / f$ noise is basically unchanged. The reasons are that $1 / f$ noise is more complicated than white noise owing to the long-range correlation properties, while the structure of white noise is simple and the main information is concentrated in the low-scale range [37]. As shown in Figure 3(a), the SDs of the RCMFDE curve and the CMFDE curve is significantly smaller than that of MFDE, indicating that sliding coarse-graining method is more stable than the traditional coarse-graining method. From Figure 3(b), we can see that the CVs (coefficients of variation) of MFDE is the largest for both white noise and $1 / f$ noise. For white noise, the CVs of RCMFDE is significantly smaller than that of CMFDE, while for noise, the CVs of the two methods is very close. However, careful observation shows that the CVs of RCMFDE is still slightly smaller than that of CMFDE in most scales. Overall, RCMFDE and CMFDE perform better than MFDE.

\section{PSO-VMD and RCMFDE-Based Fault Diagnosis Method of Hydraulic Pumps}

In order to accurately distinguish different fault states of hydraulic pumps, this paper proposes a fault diagnosis method of hydraulic pumps using PSO-VMD and RCMFDE. Figure 4 shows the flow chart of this method. It can be seen that this method is mainly divided into three parts. Firstly, PSO-VMD is adopted to remove the noise of origin vibration signals of hydraulic pumps. Secondly, RCMFDE is adopted to extract fault information. Finally, based on the obtained fault information, PSO-SVM is adopted to distinguish different work status of hydraulic pumps. The steps of this method can be summarized as follows:

Step 1: PSO-VMD is adopted to decompose collected vibration signals of hydraulic pumps, and sensitive IMF components are reconstructed according to correlation coefficient method to enhance the fault features and obtain vibration signals after noise reduction. 


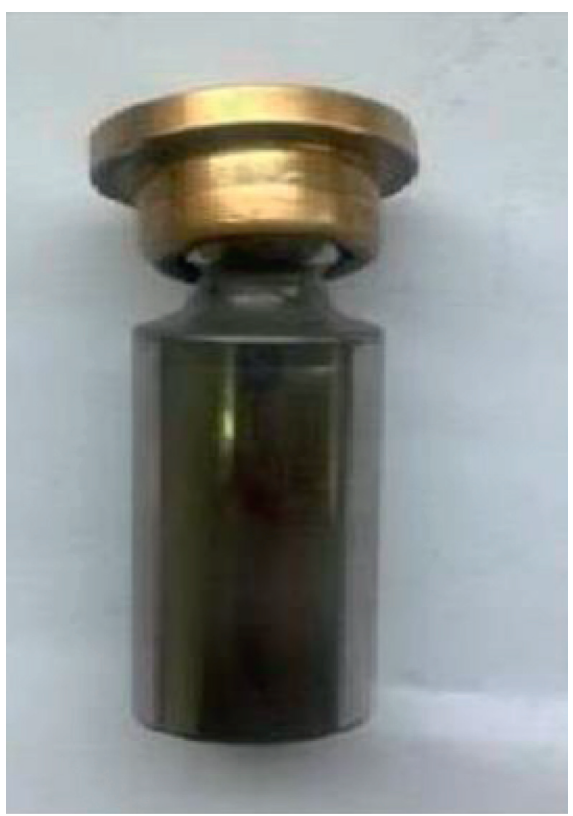

(a)

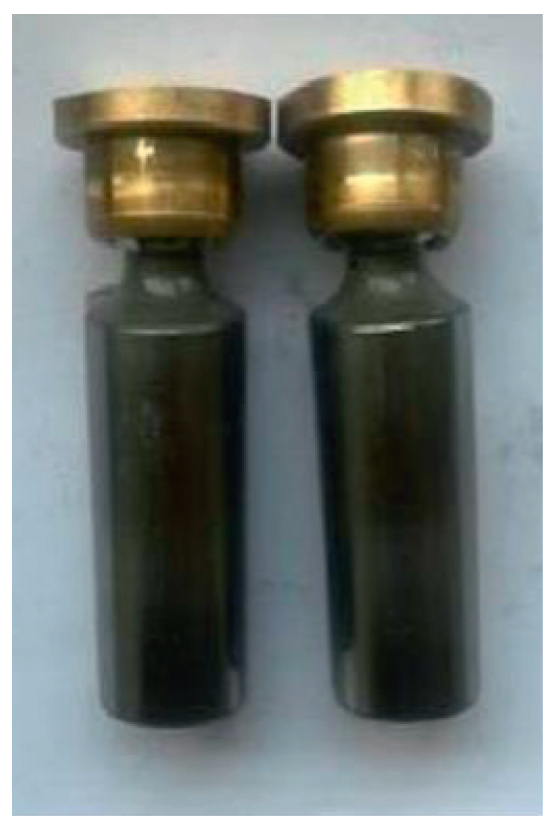

(b)

Figure 6: The fault types of plungers. (a) Plunger loose slipper. (b) Piston shoe wear.

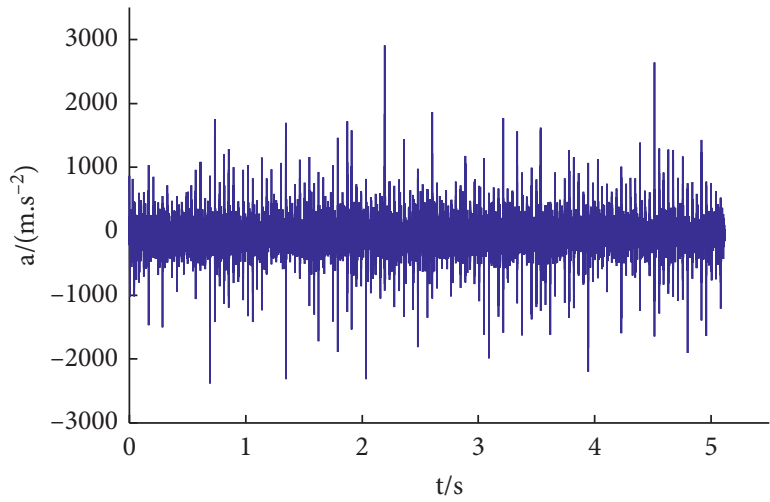

(a)

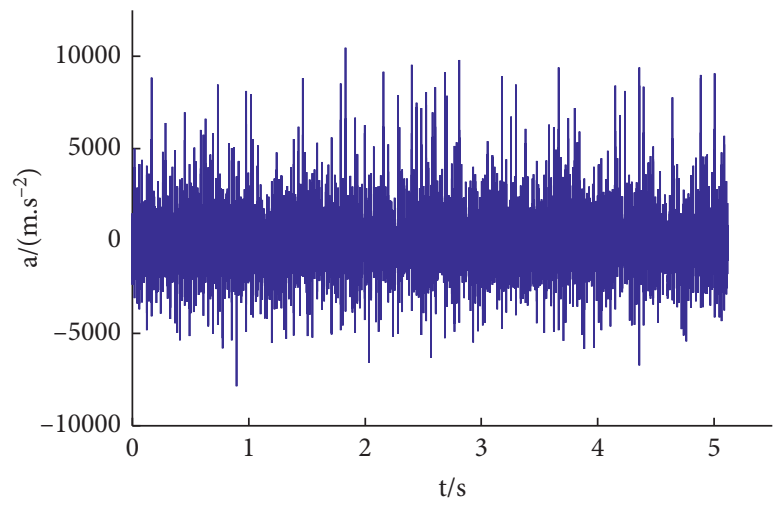

(c)

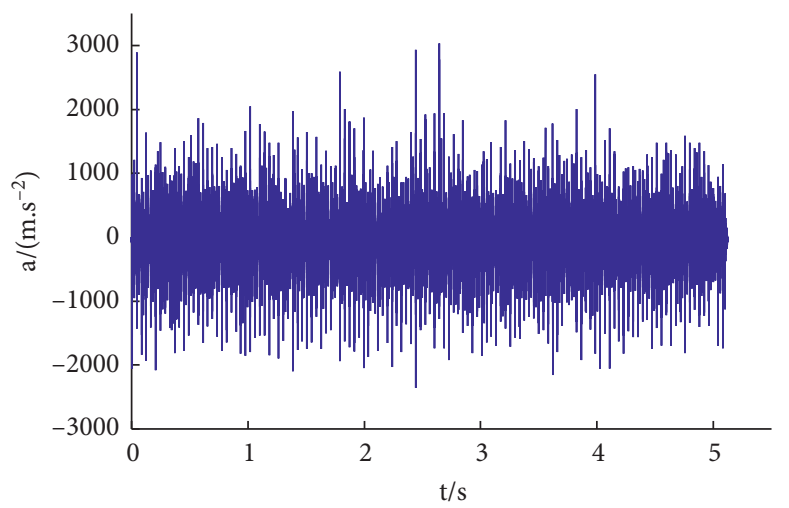

(b)

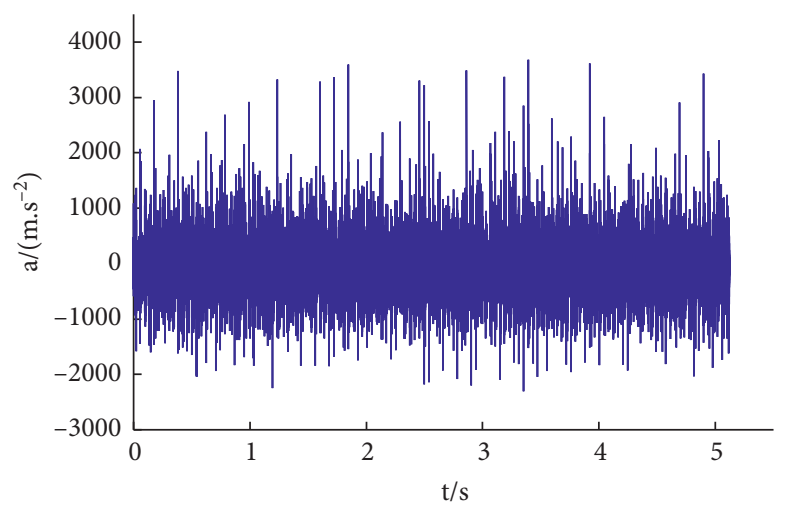

(d)

Figure 7: Time domain waveforms of original vibration signals. (a) Normal working state. (b) Single plunger loose slipper. (c) Double plungers loose slipper. (d) Piston shoe wear.

TABle 1: Parameters of PSO-VMD.

\begin{tabular}{|c|c|c|c|c|c|c|}
\hline G & $N$ & $c 1$ & $c 2$ & $w$ & $K$ & $\alpha$ \\
\hline 10 & 10 & 1.5 & 1.5 & 0.5 & {$\left[\begin{array}{ll}3 & 12\end{array}\right]$} & {$\left[\begin{array}{ll}50 & 5000\end{array}\right]$} \\
\hline
\end{tabular}




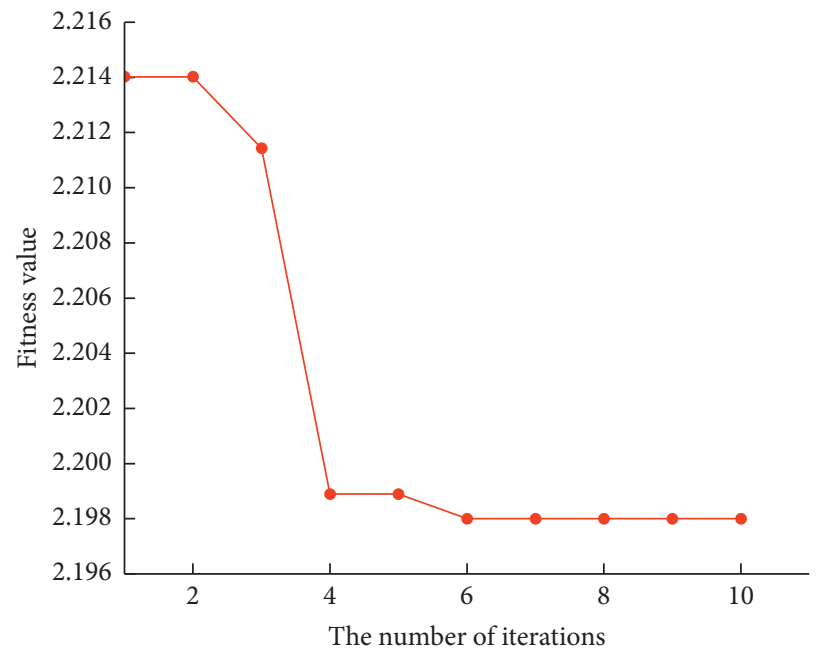

FIgURE 8: Fitness value curve.
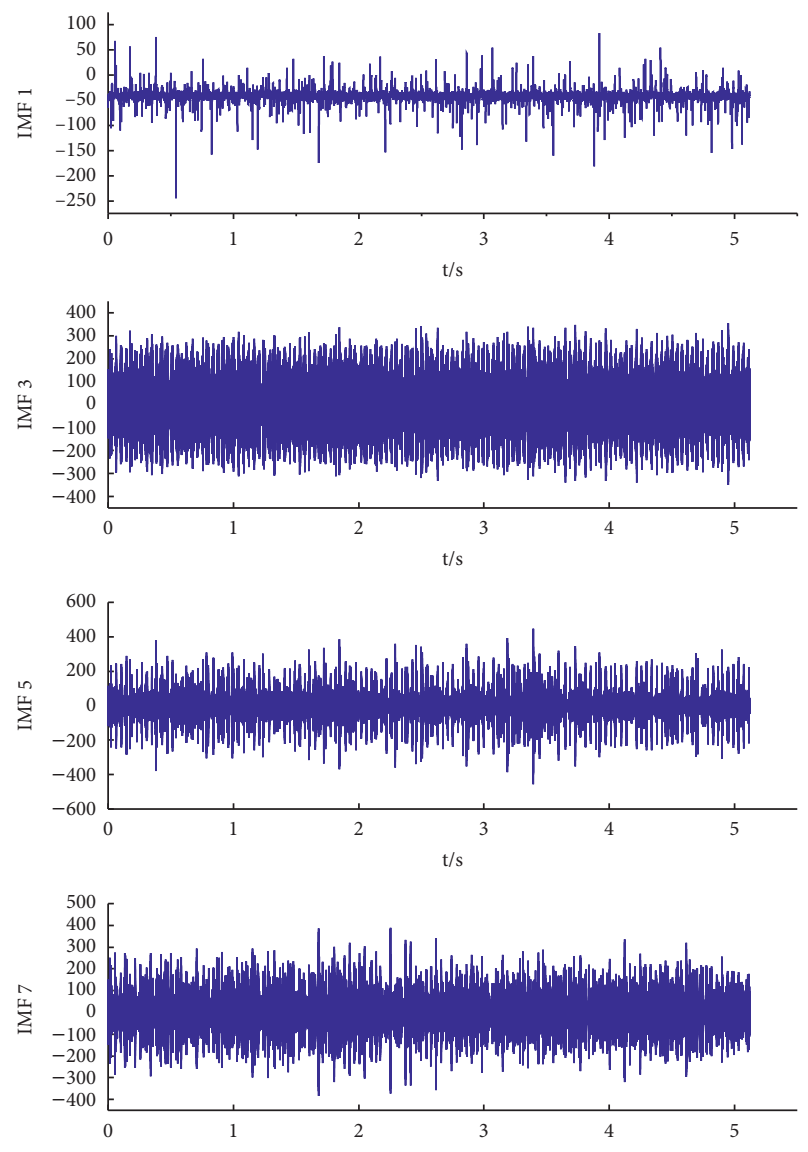
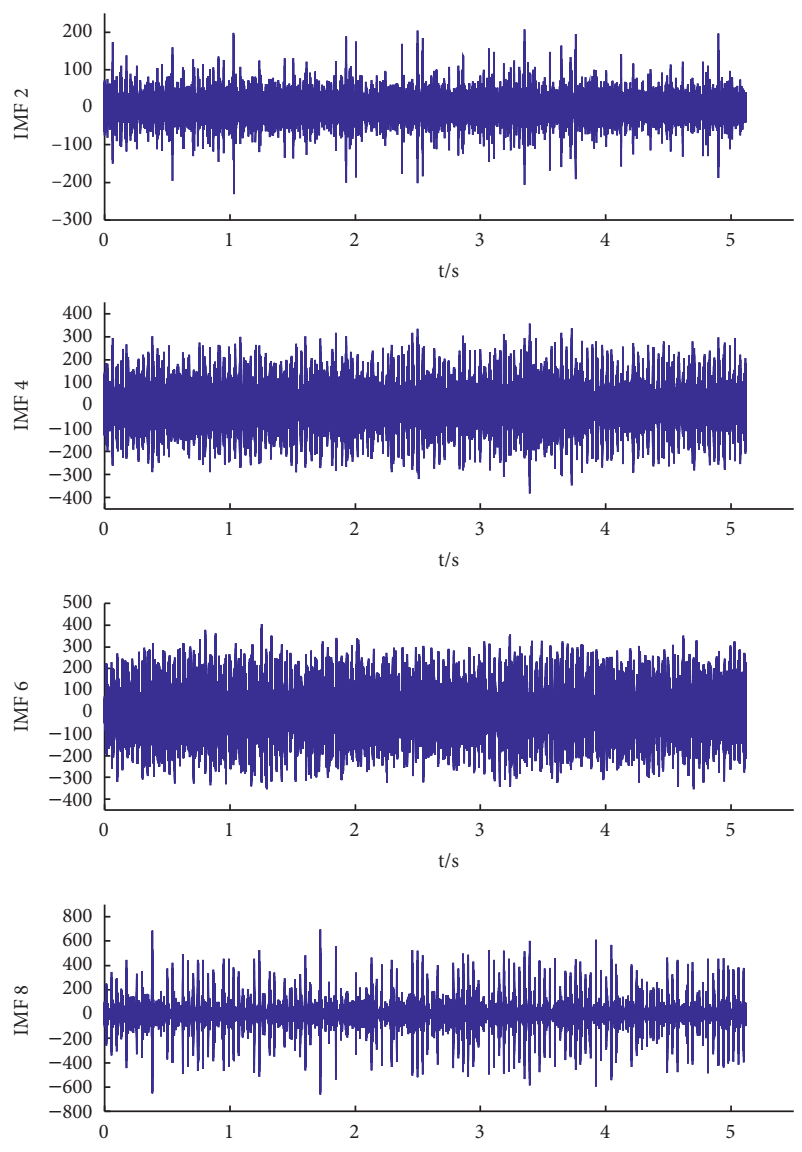

FIGURE 9: PSO-VMD decomposition.

TABle 2: Correlation coefficients.

\begin{tabular}{|c|c|c|c|c|c|c|c|c|c|}
\hline IMF & 1 & 2 & 3 & 4 & 5 & 6 & 7 & 8 & Average \\
\hline Correlation coefficient & 0.1232 & 0.1873 & 0.4628 & 0.3893 & 0.3167 & 0.4626 & 0.3734 & 0.3742 & 0.3362 \\
\hline
\end{tabular}


TABLE 3: Optimum combination of parameters.

\begin{tabular}{lc}
\hline Fault types & {$[K, \alpha]$} \\
\hline $\mathrm{N}$ & {$[10,1972]$} \\
$\mathrm{S}$ & {$[10,4820]$} \\
$\mathrm{D}$ & {$[6,4305]$} \\
$\mathrm{P}$ & {$[8,4655]$} \\
\hline
\end{tabular}

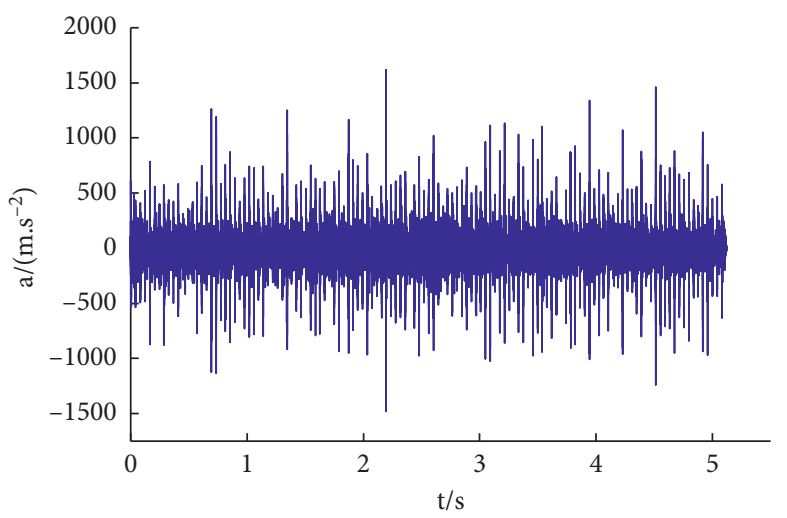

(a)

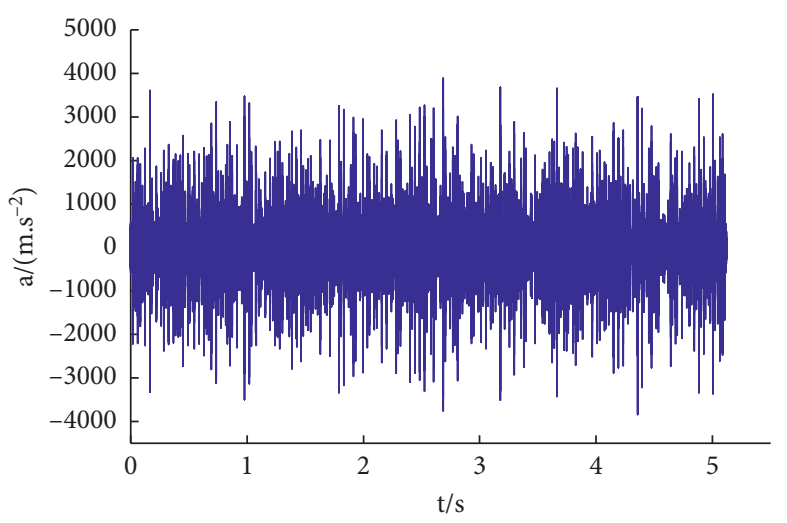

(c)

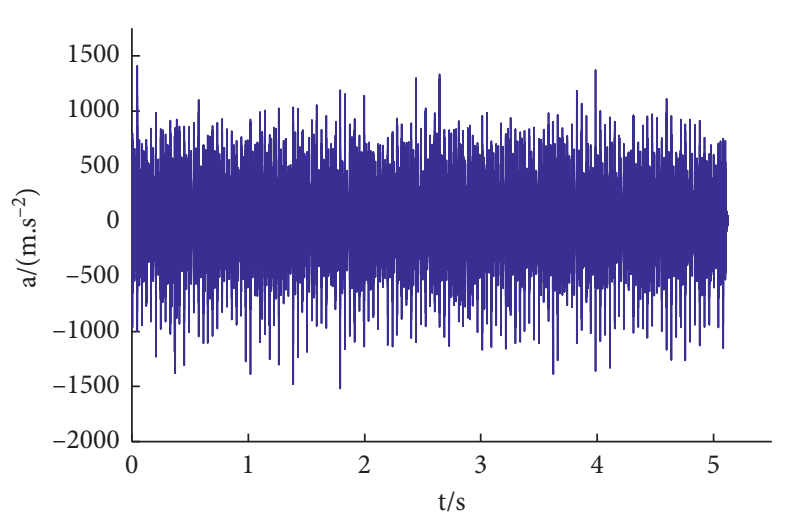

(b)

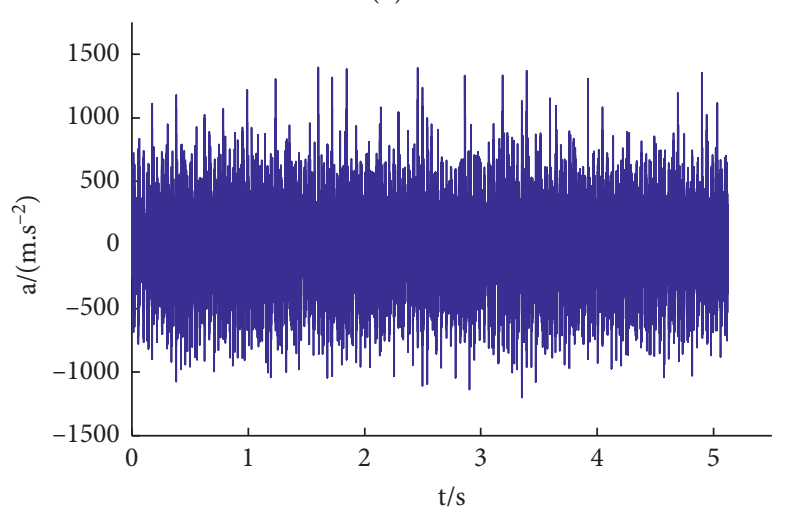

(d)

Figure 10: Time domain waveforms of denoised vibration signals. (a) Normal working state. (b) Single plunger loose slipper. (c) Double plungers loose slipper. (d) Piston shoe wear.

Step 2: take $M$ samples of vibration signals after noise reduction in each state and calculate the corresponding RCMFDE values as the fault feature vectors. In each state, fault feature vectors of $m$ samples are randomly selected as training samples, and fault feature vectors of the remaining $M-m$ samples are used as testing samples.

Step 3: all training samples are input into PSO-SVM for training.

Step 4: all testing samples are input into the fault classifier after training to identify, and complete hydraulic pumps fault identification.

\section{Experimental Validation}

5.1. Vibration Signal Collection. The hydraulic pump vibration signals were collected by the hydraulic pump experiment platform. The model type of hydraulic pump in the experiment was SY-10MCY14-1EL. Figure 5 shows the experiment device.
In the experiment, loose slipper plunger and slipper wear plunger were used to replace the normal plunger to simulate hydraulic pump faults. The plungers were shown in Figure 6. Use the $603 \mathrm{C} 01$ piezoelectricity acceleration sensor installed on the end cover of the hydraulic pump to collect the vibration signals of the hydraulic pump in different states. The sampling frequency was $20 \mathrm{kHz}$. During the experiment, the pressure of the main overflow valve was $10 \mathrm{MPa}$. The vibration signals of normal state $(\mathrm{N})$, single plunger loose slipper $(\mathrm{S})$, double plungers loose slipper (D), and piston shoes wear (P) of the hydraulic pump were collected. Figure 7 shows the signal waveforms.

5.2. Vibration Signal Noise Reduction Processing. Using PSOVMD and correlation coefficient method to remove the impact of noise, the specific steps are as follows:

(1) Use PSO to optimize VMD to get the best $[K, \alpha]$ 

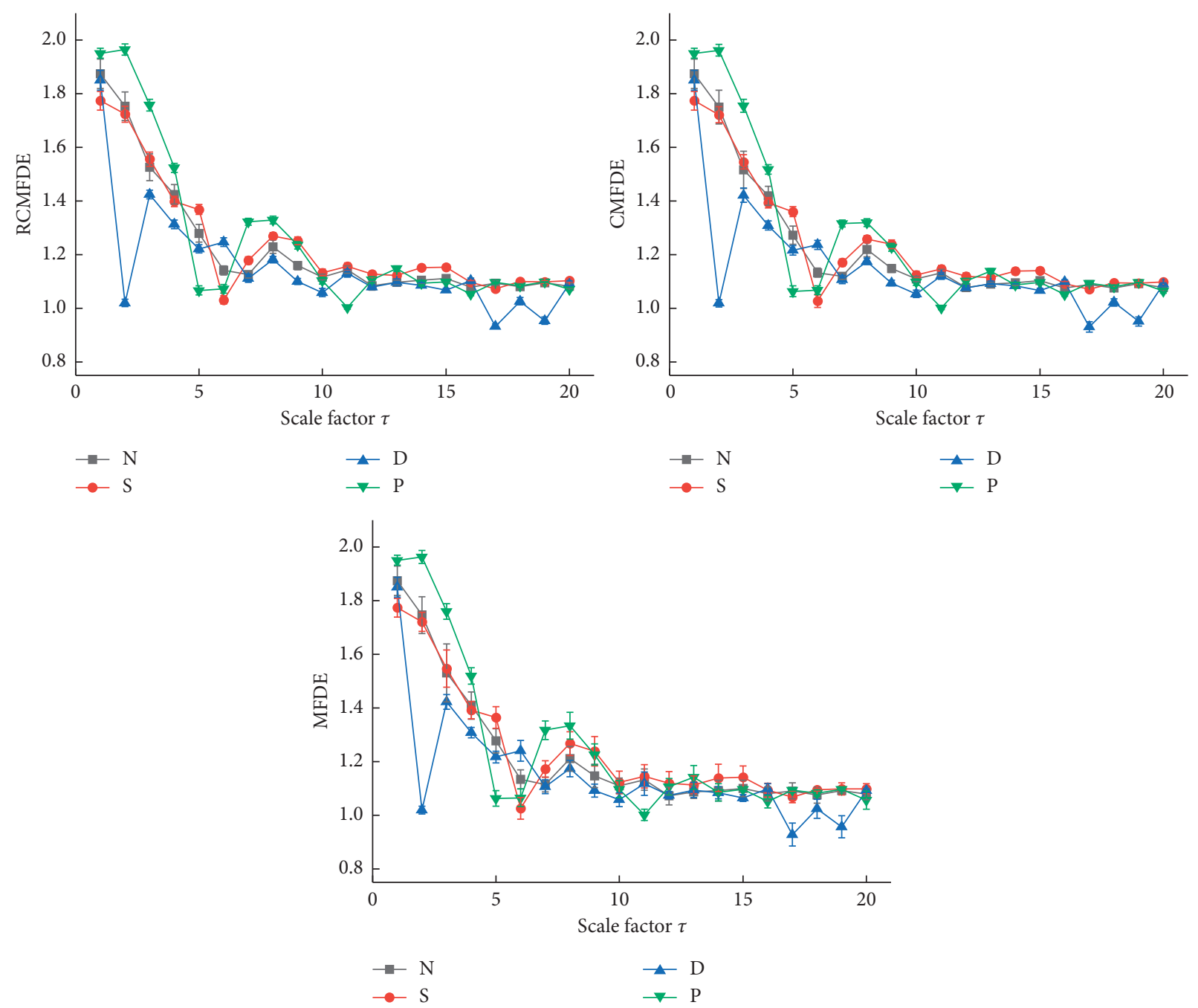

FIGURE 11: RCMFDE, CMFDE, and MFDE of four kinds states of the hydraulic pump.

(2) Calculate the correlation coefficients between the original signal and each IMF components, and calculate the mean value as the threshold values

(3) Filter out and reconstruct the IMF components whose correlation coefficient is greater than the threshold to obtain the vibration signal after noise reduction

This paper takes the vibration signal of piston shoe wear (S) as an example. First, PSO is used to optimize VMD to get the best $[K, \alpha]$. The parameters of PSO-VMD are shown in Table 1, where $G$ is the iteration number and $N$ is the particle number. Figure 8 shows the fitness curve. It can be seen that the MMPE value appears when the number of iterations is 6 , and the corresponding $[K, \alpha]$ is $[8,4655]$, which is the best $[K, \alpha]$. The waveform of $S$ vibration signal after decomposition is shown in Figure 9, and the correlation coefficients are calculated separately and shown in Table 2. Obviously, the correlation coefficients of IMF3, IMF4, IMF6, IMF7, and IMF8 are bigger than the threshold, indicating that they contain more main features of the original signal. Therefore, they can be reconstructed as sensitive IMF components to obtain the vibration signal after noise reduction. By using the same method, the denoised vibration signals of the other three states of the hydraulic pump can be obtained, respectively. Table 3 shows the corresponding $[K, \alpha]$, and Figure 10 shows the waveforms.

5.3. Fault Diagnosis for Hydraulic Pumps. After obtaining the denoised vibration signals, 50 samples are selected for each state, and the sample length is 2048 points. There are total 200 samples (50 samples $\times 4$ fault types). RCMFDE, CMFDE, and MFDE of 20 scale factors for each sample are calculated, and the corresponding results are shown in Figure 11. Obviously, the SDs of RCMFDE and CMFDE are smaller than MFDE, indicating that RCMFDE and CMFDE are more stable, but direct observation of the curves still does not distinguish the four states well.

To accurately distinguish different work states, the RCMFDE of all samples are calculated. For each state, 30 groups of samples are randomly selected for training and another 20 groups are as testing samples. Input the fault feature vectors of 120 training samples into the PSO-SVM 
TABle 4: Parameters of PSO-SVM.

\begin{tabular}{lcccccc}
\hline $\mathrm{G}$ & $N$ & $c 1$ & $c 2$ & $w$ & $c$ & $g$ \\
\hline 200 & 20 & 1.5 & 1.5 & 0.5 & {$\left[\begin{array}{ll}0 & 100\end{array}\right]$} & {$\left[\begin{array}{ll}0 & 1000\end{array}\right]$} \\
\hline
\end{tabular}

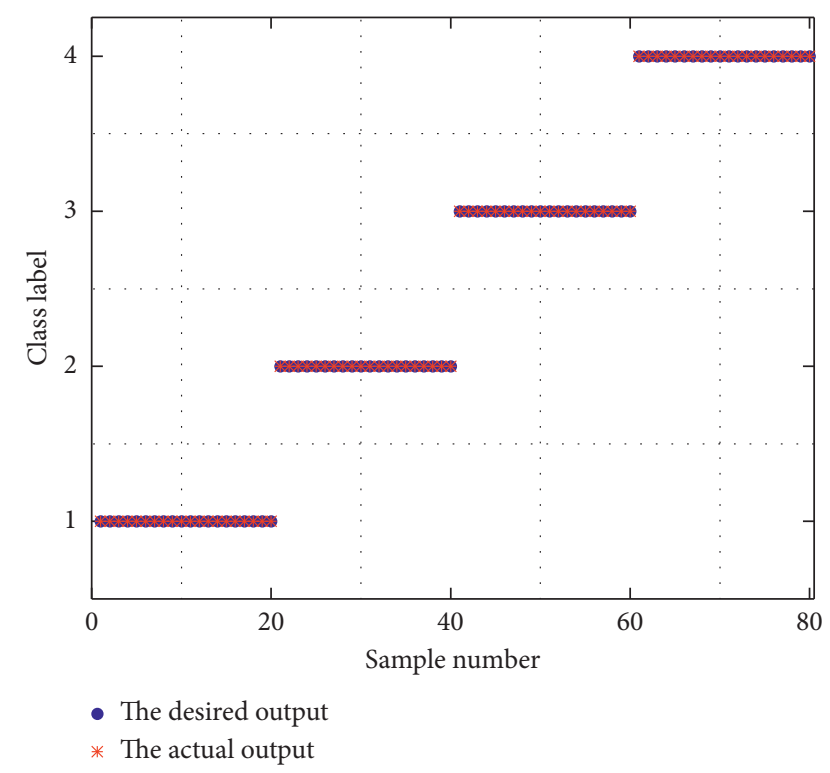

FIGURE 12: Outputs of testing samples.

TABle 5: Parameters of different methods.

\begin{tabular}{|c|c|c|c|c|c|c|}
\hline & RCMFDE & CMFDE & MFDE & MDE & MFE & MSE \\
\hline $\begin{array}{l}\text { Embedding } \\
\text { dimension }\end{array}$ & 2 & 2 & 2 & 2 & 2 & 2 \\
\hline Time delay & 1 & 1 & 1 & 1 & 1 & 1 \\
\hline Classes & 6 & 6 & 6 & 6 & & \\
\hline Threshold & & & & & 0.15 & 0.15 \\
\hline $\begin{array}{l}\text { Fuzzy } \\
\text { parameter }\end{array}$ & & & & & 2 & \\
\hline $\begin{array}{l}\text { Largest scale } \\
\text { factor }\end{array}$ & 20 & 20 & 20 & 20 & 20 & 20 \\
\hline
\end{tabular}

classifier for training. The labels corresponding to the four states of $\mathrm{N}, \mathrm{S}, \mathrm{D}$, and $\mathrm{P}$ are $1,2,3$, and 4, respectively. The parameter settings of PSO-SVM are shown in Table 4, where $c$ and $g$ are, respectively, penalty parameter and kernel function of SVM. Then, the fault feature vectors of 80 testing samples are input into PSO-SVM for recognition and classification. As shown in Figure 12, the method proposed can well distinguish the four states of the hydraulic pump, and the accuracy is $100 \%$.

To compare the performance of different methods, CMFDE, MFDE, MDE, MFE, and MSE are adopted to replace the RCMFDE for the hydraulic pump fault diagnosis. Each method has been run 50 times. Table 5 shows the parameters setting $[24,26]$. The results are shown in Figure 13 and Table 6, where CPU time in Table 6 refers to the time it takes to calculate the entropy of all samples. It can be seen that the calculation efficiency and accuracy of MFDE and MDE are obviously higher than those of MFE and MSE. It verifies the superiority of $\mathrm{DE}$ when compared with $\mathrm{SE}$ and

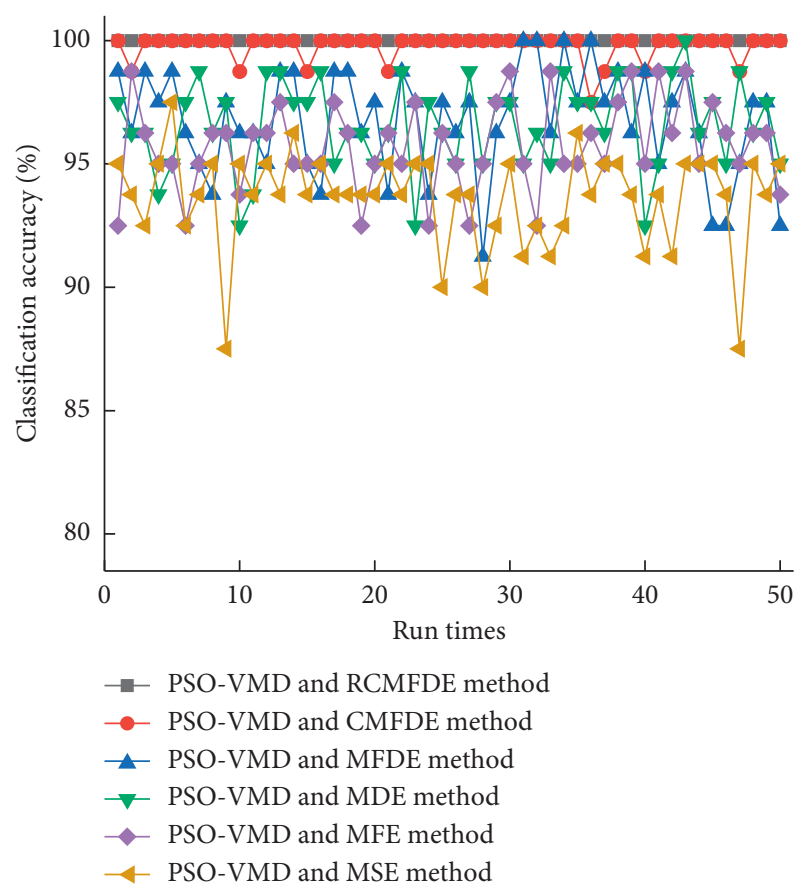

FIgURE 13: Classification accuracy of different methods.

TABLE 6: The accuracy of different methods.

\begin{tabular}{lcccc}
\hline \multirow{2}{*}{ Different methods } & \multicolumn{3}{c}{ Classification } & \\
& Maccuracy (\%) & CPU time (s) \\
& Min & Mean & \\
\hline PSO-VMD and RCMFDE & 100 & 100 & 100 & 204.01 \\
PSO-VMD and CMFDE & 100 & 98.75 & 99.78 & 205.88 \\
PSO-VMD and MFDE & 100 & 91.25 & 96.75 & 39.12 \\
PSO-VMD and MDE & 98.75 & 92.50 & 96.58 & 39.29 \\
PSO-VMD and MFE & 98.75 & 92.50 & 95.73 & 843.11 \\
PSO-VMD and MSE & 97.5 & 87.5 & 93.63 & 453.50 \\
\hline
\end{tabular}

FE. Meanwhile, the calculation efficiency of RCMFDE and CMFDE is basically the same and is lower than MFDE and MDE, which can be interpreted as the coarse graining processing and the entropy values calculation processing of RCMFDE and CMFDE are more complicated, so it takes more time. However, the accuracy of these two methods is obviously higher, especially the proposed method. The accuracy of 50 test results is all $100 \%$, which proves that this method can well distinguish different fault states of the hydraulic pump.

To compare the effect of fault identification before and after noise reduction, without PSO-VMD, multiscale entropy methods are adopted to directly extract fault information of the hydraulic pump. The sample processing method is the same as previous. Figure 14 and Table 7 show the final results. We can see that without using PSO-VMD, the accuracy of all methods decreases obviously, while RCMFDE still has the highest accuracy, indicating that RCMFDE has a stronger ability to extract fault features. The comparison proves that PSO-VMD and the correlation coefficient method can highlight the fault characteristics and have a good noise reduction effect. 


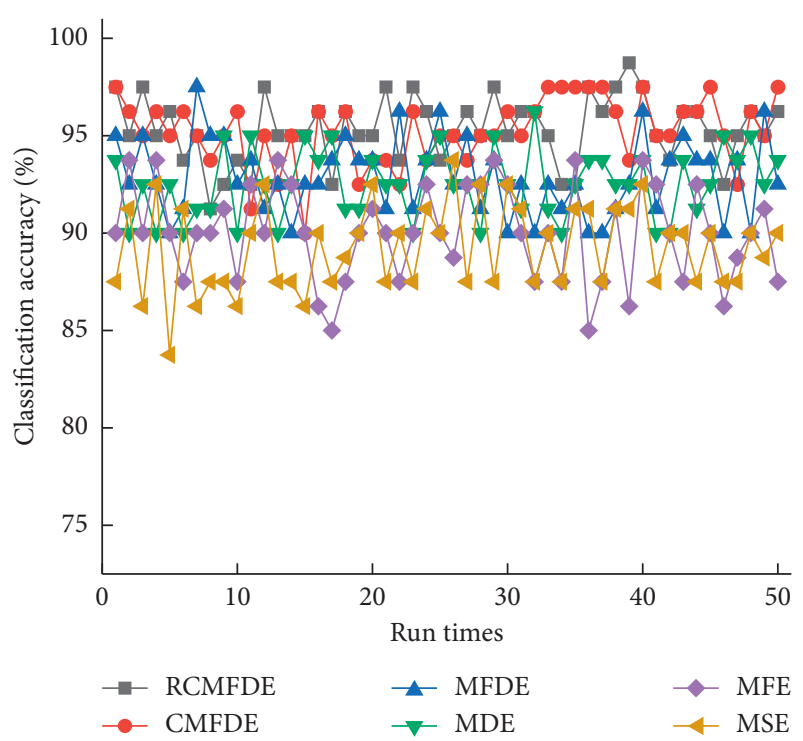

FIGURE 14: Classification accuracy of different methods without PSO-VMD.

TABLE 7: The accuracy of different methods without PSO-VMD.

\begin{tabular}{lccc}
\hline \multirow{2}{*}{ Different methods } & \multicolumn{3}{c}{ Classification accuracy (\%) } \\
& Max & Min & Mean \\
\hline RCMFDE & 98.75 & 91.25 & 95.33 \\
CMFDE & 97.5 & 90 & 95.25 \\
MFDE & 97.5 & 90 & 92.90 \\
MDE & 95 & 90 & 92.53 \\
MFE & 93.75 & 86.25 & 90.03 \\
MSE & 93.75 & 83.75 & 89.25 \\
\hline
\end{tabular}

\section{Conclusions}

In order to accurately and efficiently identify different fault states of hydraulic pumps to prevent the occurrence of safety accidents. This paper proposes a new method for hydraulic pumps fault diagnosis based on PSO-VMD and RCMFDE. Experimental analysis proves the great performance of the proposed method. The main work and innovations of this article are as follows:

(1) The coarse-graining method of MFDE is improved and RCMFDE is proposed, which has better fault features extraction ability and stability.

(2) PSO is used to optimize VMD, which solves the defect that the parameters need to be set manually.

(3) Combining PSO-VMD and RCMFDE, a new hydraulic pump fault diagnosis method is proposed. Experimental analysis shows that this method has the best fault diagnosis capability compared with other methods.

In the future, we will further study the entropy theory and try to apply similar methods to fault diagnosis of other mechanical equipment such as planetary gearboxes and rolling bearings, so as to expand the application scope of similar methods.

\section{Data Availability}

The experimental data are provided by the Mechanical Engineering College and cannot be disclosed.

\section{Conflicts of Interest}

The authors declare that there are no conflicts of interest regarding the publication of this paper.

\section{Acknowledgments}

Thanks are due to the Mechanical Engineering College for supplying the hydraulic pump data.

\section{References}

[1] L. Guo, Y. Lei, N. Li, T. Yan, and N. Li, "Machinery health indicator construction based on convolutional neural networks considering trend burr," Neurocomputing, vol. 292, pp. 142-150, 2018.

[2] R. Zhou, "Current research and developing trends on fault diagnosis of hydraulic systems," Chinese Journal of Mechanical Engineering, vol. 42, no. 09, pp. 6-14, 2006.

[3] H. Zhao, H. Liu, J. Xu, and W. Deng, "Performance prediction using high-order differential mathematical morphology gradient spectrum entropy and extreme learning machine," IEEE Transactions on Instrumentation and Measurement, vol. 69, pp. 4165-4172, 2020.

[4] J. Chen and Z. Chen, "Present situation and development trend of failure diagnosis technology of the hydraulic system," Machine Tool \& Hydraulics, vol. 10, 2008.

[5] Z. He, J. Chen, T. Wang, and F. Chu, Theories and Applications of Machinery Fault Diagnostics, Higher Education Press, Beijing, China, 2010.

[6] W. Deng, H. Liu, J. Xu, H. Zhao, and Y. Song, "An improved quantum-inspired differential evolution algorithm for deep belief network," IEEE Transactions on Instrumentation and Measurement, p. 1, 2020.

[7] Z. Zhao, M. Jia, F. Wang, and S. Wang, "Intermittent chaos and sliding window symbol sequence statistics-based early fault diagnosis for hydraulic pump on hydraulic tube tester," Mechanical Systems and Signal Processing, vol. 23, no. 5, pp. 1573-1585, 2009.

[8] Y. Lei, J. Lin, Z. He, and M. J. Zuo, “A review on empirical mode decomposition in fault diagnosis of rotating machinery," Mechanical Systems \& Signal Processing, vol. 35, no. 1-2, pp. 108-126, 2013.

[9] S. Dong, B. Tang, and R. Chen, "Bearing running state recognition based on non-extensive wavelet feature scale entropy and support vector machine," Measurement, vol. 46, no. 10, pp. 4189-4199, 2013.

[10] J. Cheng, Y. Yang, and Y. Yang, "A rotating machinery fault diagnosis method based on local mean decomposition," Digital Signal Processing, vol. 22, no. 2, pp. 356-366, 2012.

[11] K. Dragomiretskiy and D. Zosso, "Variational mode decomposition," IEEE Transactions on Signal Processing, vol. 62, no. 3, pp. 531-544, 2014.

[12] T. Zan, Z. Pang, M. Wang, and X. Gao, "Research on early fault diagnosis of rolling bearing based on VMD," in Proceedings of the 2018 6th International Conference on Mechanical, Automotive and Materials Engineering (CMAME), pp. 41-45, Hong Kong, China, August 2018. 
[13] D. Song, C. Lu, and J. Ma, "Gearbox fault diagnosis based on VMD-MSE and adaboost classifier," Vibroengineering Procedia, vol. 14, pp. 120-125, 2017.

[14] D. Whitley, "A genetic algorithm tutorial," Statistics and Computing, vol. 4, pp. 65-85, 1994.

[15] W. Deng, J. Xu, and H. Zhao, "An improved ant colony optimization algorithm based on hybrid strategies for scheduling problem," IEEE Access, vol. 7, pp. 20281-20292, 2019.

[16] J. Kennedy and R. Eberhart, "Particle swarm optimization," in Proceedings of ICNN'95-International Conference on Neural Networks, vol. 4, pp. 1942-1948, Perth, Australia, August 1995.

[17] J. S. Richman and J. R. Moorman, "Physiological time-series analysis using approximate entropy and sample entropy," American Journal of Physiology-Heart and Circulatory Physiology, vol. 278, no. 6, pp. H2039-H2049, 2000.

[18] Z. Zhao and S. Yang, "Sample entropy-based roller bearing fault diagnosis method," Journal of Vibration and Shock, vol. 31, pp. 136-140, 2012.

[19] C. Bandt and B. Pompe, "Permutation entropy: a natural complexity measure for time series," Physical Review Letters, vol. 88, p. 17, 2002.

[20] R. Yan, Y. Liu, and R. X. Gao, "Permutation entropy: a nonlinear statistical measure for status characterization of rotary machines," Mechanical Systems and Signal Processing, vol. 29, pp. 474-484, 2012.

[21] B. Kosko, "Fuzzy entropy and conditioning," Information Sciences, vol. 40, no. 2, pp. 165-174, 1986.

[22] Z. Jinde, C. Junsheng, and Y. Yang, "A rolling bearing fault diagnosis approach based on LCD and fuzzy entropy," Mechanism and Machine Theory, vol. 70, pp. 441-453, 2013.

[23] M. Costa, A. L. Goldberger, and C. K. Peng, "Multiscale entropy analysis of complex physiologic time series," Physical Review Letters, vol. 89, pp. 705-708, 2007.

[24] W. Yu, M. Xu, Y. Li, and W. Huang, "A feature extraction method based on ICD and MSE for gearbox," Journal of Vibroengineering, vol. 18, pp. 3596-3607, 2016.

[25] S.-D. Wu, P.-H. Wu, C.-W. Wu, J.-J. Ding, and C.-C. Wang, "Bearing fault diagnosis based on multiscale permutation entropy and support vector machine," Entropy, vol. 14, no. 8, pp. 1343-1356, 2012.

[26] J. Zheng, J. Cheng, Y. Yang, and S. Luo, "A rolling bearing fault diagnosis method based on multi-scale fuzzy entropy and variable predictive model-based class discrimination," Mechanism and Machine Theory, vol. 78, pp. 187-200, 2014.

[27] S.-D. Wu, C.-W. Wu, and A. Humeau-Heurtier, "Refined scale-dependent permutation entropy to analyze systems complexity," Physica A: Statistical Mechanics and Its Applications, vol. 450, pp. 454-461, 2016.

[28] B. Fadlallah, B. Chen, A. Keil, and J. Príncipe, "Weightedpermutation entropy: a complexity measure for time series incorporating amplitude information," Physical Review E, vol. 87, no. 2, 2013.

[29] H. Azami and J. Escudero, "Refined composite multivariate generalized multiscale fuzzy entropy: a tool for complexity analysis of multichannel signals," Physica A: Statistical Mechanics and Its Applications, vol. 465, pp. 261-276, 2017.

[30] M. Rostaghi and H. Azami, "Dispersion entropy: a measure for time series analysis," IEEE Signal Processing Letters, vol. 23, no. 5, pp. 610-614, 2016.

[31] H. Azami and J. Escudero, "Amplitude- and fluctuation-based dispersion entropy,” Entropy, vol. 20, no. 3, p. 210, 2018.
[32] H. Azami, S. E. Arnold, S. Sanei et al., "Multiscale fluctuationbased dispersion entropy and its applications to neurological diseases," IEEE Access, vol. 7, pp. 68718-68733, 2019.

[33] S.-D. Wu, C.-W. Wu, K.-Y. Lee, and S.-G. Lin, "Modified multiscale entropy for short-term time series analysis," Physica A: Statistical Mechanics and Its Applications, vol. 392, no. 23, pp. 5865-5873, 2013.

[34] X. Gan, H. Lu, and G. Yang, "Fault diagnosis method for rolling bearings based on composite multiscale fluctuation dispersion entropy," Entropy, vol. 21, 2019.

[35] H. Zhao, J. Zheng, W. Deng, and Y. Song, "Semi-supervised broad learning system based on manifold regularization and broad network," IEEE Transactions on Circuits and Systems I: Regular Papers, vol. 67, no. 3, pp. 983-994, 2020.

[36] S. Wang, L. Dong, J. Wang, H. Wang, C. Ji, and J. Hong, "Experiment study on small leak detection and diagnosis for propulsion system pipelines of sounding rocket," IEEE Access, vol. 8, pp. 8743-8753, 2020.

[37] Y. Xiaoan and J. Minping, "Intelligent fault diagnosis of rotating machinery using improved multiscale dispersion entropy and mRMR feature selection," Knowledge Based Systems, vol. 163, pp. 450-471, 2019. 